EFI-05-15

\title{
Boundary Ground Ring in Minimal String Theory
}

\author{
Anirban Basu ${ }^{1}$ and Emil J. Martinec ${ }^{2}$ \\ Enrico Fermi Institute and Department of Physics, University of Chicago, \\ 5640 S. Ellis Avenue, Chicago IL 60637, USA
}

\begin{abstract}
We obtain relations among boundary states in bosonic minimal open string theory using the boundary ground ring. We also obtain a difference equation that boundary correlators must satisfy.
\end{abstract}

\footnotetext{
${ }^{1}$ email: basu@theory.uchicago.edu, Address after Sep 11, 2005: School of Natural Sciences, Institute for Advanced Study, Princeton, NJ 08540, USA

${ }^{2}$ email: ejm@theory.uchicago.edu
} 


\section{Introduction}

Exactly solvable string theories are of intrinsic interest, and have also proven useful in elucidating the structure of string theory in general. The basic idea is to consider twodimensional gravity coupled to $c_{M} \leq 1$ matter conformal field theory. It is well-known that the physical states of this theory carry non-trivial ghost numbers [1], and correspond to operators which are non-trivial in the BRST cohomology of the theory. Among these operators, those with ghost number zero and conformal dimension zero form the ground ring of the theory, where ring multiplication corresponds to OPE in the field theory [2], modulo BRST exact operators. Both the closed and the open string sectors contain ground ring operators in their spectrum. In this work, we shall analyze the role of the boundary ground ring in minimal (bosonic) string theory.

\subsection{Summary of minimal string theory}

To begin, we briefly review certain facts about minimal string theory which will be useful later. The world-sheet theory of two-dimensional gravity is given by Liouville conformal field theory [3], which we couple to "matter" CFT to define minimal string theory. Consider Liouville theory with background charge $Q_{L}=b+1 / b$, where $b$ is the coupling. In addition to the kinetic term and the curvature coupling specifying the background charge, the other terms in the action are

$$
\mu \int_{M} d^{2} x \sqrt{g} e^{2 b \phi}+\mu_{B} \int_{\partial M} d y g^{1 / 4} e^{b \phi},
$$

where $\mu$ and $\mu_{B}$ are the bulk and boundary cosmological constants respectively. The first term corresponds to the bulk Liouville interaction, while the second yields the boundary interaction. The central charge of this CFT is given by

$$
c_{L}=1+6 Q_{L}^{2}
$$

Since we shall be considering boundary operators in this theory, we need to know their conformal dimensions. The boundary operator $e^{\beta \phi}$ has conformal dimension $\beta\left(Q_{L}-\beta\right)$. In particular, $\Delta\left(e^{b \phi}\right)=1$ and so the boundary interaction is a marginal deformation. We want to couple this theory to matter CFT with background charge $Q_{M}$. The central charge of the matter CFT is

$$
c_{M}=1-6 Q_{M}^{2} .
$$


On coupling Liouville theory to matter, we require that $c_{L}+c_{M}=26$, which leads to $Q_{M}^{2}=(b-1 / b)^{2}$. In particular, for the matter CFT we shall consider the $(p, q)$ minimal models [4] where $p$ and $q$ are relatively prime integers, and $p>q$. Thus we get that $b-1 / b= \pm(\sqrt{p / q}-\sqrt{q / p})$. Since $b$ is the coupling we take it to be positive, so that the theory is free as $\phi \rightarrow-\infty$. This tells us that

$$
b=\sqrt{\frac{q}{p}}
$$

Then the boundary cosmological constant term $e^{b \phi}$ satisfies the Seiberg bound $b<Q_{L} / 2[5$, $6] .^{3}$

In the matter CFT, the boundary vertex operators are given by $e^{i \alpha_{r, s} X}$ where

$$
\alpha_{r, s}=\frac{1}{2}(1-r) \alpha_{+}+\frac{1}{2}(1-s) \alpha_{-},
$$

and $\alpha_{+}=\sqrt{p / q}, \alpha_{-}=-\sqrt{q / p}$. They have conformal dimension

$$
\Delta\left(e^{i \alpha_{r, s} X}\right)=\alpha_{r, s}\left(\alpha_{r, s}-Q_{M}\right)
$$

There are $(p-1)(q-1) / 2$ such vertex operators having $1 \leq r<q, 1 \leq s<p$ and $p r-q s>0$, which fill out the Kac table of degenerate operators (c.f. [8]). Using

$$
Q_{M}=\frac{1}{b}-b=\sqrt{\frac{p}{q}}-\sqrt{\frac{q}{p}}
$$

we see that

$$
\Delta\left(e^{i \alpha_{r, s} X}\right)=\frac{(p r-q s)^{2}-(p-q)^{2}}{4 p q},
$$

which is the Kac formula.

Open string boundary conditions for the matter also come in one-to-one correspondence with the Kac table [9], and are thus labelled by integers $(m, n), 1 \leq m<q, 1 \leq n<p$. In the Liouville field theory, the parameter $\mu_{B}$ enters the boundary condition through the boundary terms in the variation of the action. It proves more convenient to express the boundary parameter in terms of the uniformization parameter $\sigma$ defined by [10]

$$
\frac{\mu_{B}^{2}}{\mu} \sin \pi b^{2}=\cosh ^{2} \pi b \sigma .
$$

Boundary states describe D-brane boundary conditions from the perspective of the dual closed string channel, and are also labelled by $(\sigma ; m, n)$.

\footnotetext{
${ }^{3}$ The other choice $b=\sqrt{p / q}$ violates the bound but is a distinct, allowed operator [7].
} 
Unlike the closed string vertex operators, in order to specify open string vertex operators we also need to specify the boundary conditions across their insertion points on the boundary. So we need to specify the values of $\mu_{B}$ in the Liouville sector, and $(m, n)$ (where $1 \leq m<q$ and $1 \leq n<p)$ in the matter sector, on either side of the insertion of the vertex operator on the boundary. So we shall express boundary vertex operators as ${ }_{I_{L}}^{\sigma_{L}}[\mathcal{O}]_{I_{R}}^{\sigma_{R}}$, where $\sigma_{L}\left(\sigma_{R}\right)$ are the values of $\sigma$ on the left (right) of the insertion, and $I_{L}\left(I_{R}\right)^{4}$ are the values of $(m, n)$ on the left (right) of the insertion. Let us now mention the constraints on the choice of these boundary conditions, which depend on the structure of degenerate representations of the two CFTs.

In the matter sector, if the boundary vertex operator contains a degenerate primary of the Virasoro algebra with Kac label $(r, s)$, then the constraint is given by

$$
(r, s) \times\left(m_{L}, n_{L}\right) \sim\left(m_{R}, n_{R}\right),
$$

or in other words, $\left(m_{R}, n_{R}\right)$ should be in the minimal model fusion rule of $(r, s)$ and $\left(m_{L}, n_{L}\right)[9,11]$.

In the Liouville sector, in order to know if there is any relation between $\sigma_{L}$ and $\sigma_{R}$, we need to know the structure of the degenerate representations of the Liouville Virasoro algebra. From the expression for the Kac determinant, it follows that the degenerate representations of the Liouville Virasoro algebra have conformal primaries $e^{\gamma_{r, s} \phi}$ where

$$
\gamma_{r, s}=\frac{(1-r) p+(1-s) q}{2 \sqrt{p q}}
$$

where $r, s \geq 1$.

In boundary Liouville theory, vertex operators containing degenerate Liouville primaries satisfying (11) have constraints between $\sigma_{L}$ and $\sigma_{R}[10,12]$. For example, for the degenerate primary $e^{-b \phi / 2}$ the restriction is that $\sigma_{L}-\sigma_{R}= \pm i b$ or $\sigma_{L}+\sigma_{R}= \pm i b$. (It follows that the condition for $e^{-\phi / 2 b}$ to be a degenerate primary is that $\sigma_{L}-\sigma_{R}= \pm i / b$ or $\sigma_{L}+\sigma_{R}= \pm i / b$.) Thus $\sigma$ changes along the boundary across the insertion point. However, at the next level, for $e^{-b \phi}$ to be a degenerate primary, one of the possible choices is $\sigma_{L}=\sigma_{R}$ (this is also true for $\left.e^{-\phi / b}\right)$. So in this case $\sigma$ does not change along the boundary. In general for the degenerate primaries $e^{-n b \phi}$ and $e^{-n \phi / b}$, where $n$ is a non-negative integer, $\sigma_{L}=\sigma_{R}$ is an allowed choice.

\footnotetext{
${ }^{4} I_{i} \equiv\left(m_{i}, n_{i}\right)$
} 
The open string tachyon and ground ring vertex operators will be relevant to our discussion. The open string tachyon vertex operators are given by

$$
T_{r, s}=c e^{i \alpha_{r, s} X} e^{\beta_{r, s} \phi}
$$

where we have dropped the indices $\sigma_{L}, \sigma_{R}, I_{L}$ and $I_{R}$ for brevity. Demanding $\Delta\left(T_{r, s}\right)=0$, we get that

$$
\beta_{r, s}=\frac{p(1-r)+q(1+s)}{2 \sqrt{p q}} .
$$

In what follows we will not be considering the other Liouville dressing (the one that violates the Seiberg bound).

\subsection{The boundary ground ring}

Let us next consider the elements of the boundary ground ring [13]. A generic element of the ground ring is given by ${ }_{I_{L}}^{\sigma_{L}}\left[\mathcal{O}_{r, s}\right]_{I_{R}}^{\sigma_{R}}$, where $1 \leq r<q$ and $1 \leq s<p$ and so there are $(p-1)(q-1)$ ring elements. To construct ${ }_{I_{L}}^{\sigma_{L}}\left[\mathcal{O}_{r, s}\right]_{I_{R}}^{\sigma_{R}}$, we first consider ${ }_{I_{L}}^{\sigma_{L}}\left[V_{r, s}^{(L)} V_{r, s}^{(M)}\right]_{I_{R}}^{\sigma_{R}}$, where ${ }^{\sigma_{L}}\left[V_{r, s}^{(L)}\right]^{\sigma_{R}}$ and ${ }_{I_{L}}\left[V_{r, s}^{(M)}\right]_{I_{R}}$ are Liouville and matter degenerate primaries respectively. More explicitly,

$$
V_{r, s}^{(L)}=e^{\gamma_{r, s} \phi}
$$

where $\gamma_{r, s}$ is given by (11), and

$$
V_{r, s}^{(M)}=e^{i \alpha_{r, s} X}
$$

where $\alpha_{r, s}$ is given by (15). Now it is easy to check that $\Delta\left(V_{r, s}^{(L)} V_{r, s}^{(M)}\right)=1-r s$. So ${ }_{I_{L}}^{\sigma_{L}}\left[\mathcal{O}_{r, s}\right]_{I_{R}}^{\sigma_{R}}$ is obtained by acting on ${ }_{I_{L}}^{\sigma_{L}}\left[V_{r, s}^{(L)} V_{r, s}^{(M)}\right]_{I_{R}}^{\sigma_{R}}$ with $\mathcal{L}_{r, s}$ having conformal dimension $r s-1$ and ghost number zero. $\mathcal{L}_{r, s}$ is constructed entirely out of the $b c$ ghosts, and polynomials in $L_{-n}(n \geq 1)$ for both Liouville and matter. The boundary ground ring is generated by the boundary ground ring elements $\mathcal{O}_{1,2}$ and $\mathcal{O}_{2,1}$, since according to the fusion rules these contain all other ground ring elements in their products. We will not address the issue of the ring structure here, apart from some comments in the final section.

The bulk tachyon operators form a module under the action of the ground ring; similarly the boundary tachyons constitute a module under the action of the boundary ground ring. Furthermore, the boundary ground ring contains elements which change the boundary conditions in both matter and Liouville; thus the boundary conditions themselves are acted on by the boundary ground ring, and we can use this fact to deduce relations among various boundary conditions. 


\subsection{The rest of the paper}

In this work, we study the role of the boundary ground ring in minimal string theory. To begin, in section 2 we argue that the disc one-point function with only one boundary insertion and no bulk insertions vanishes unless the boundary vertex operator is the identity (and the correlator is the partition function). In particular, this holds for the case when the one-point function is any boundary ground ring operator. This auxilliary result is a necessary ingredient for the subsequent manipulations in section 3. There, we compute disc partition functions with two boundary ground ring insertions and no bulk insertions in two different ways, yielding relations among partition functions, which we postulate to lead to an exact relation (at the level of BRST cohomology) among boundary states in the full theory:

$$
|\sigma ; j, k\rangle+|\sigma ; j, k-2\rangle=|\sigma+i b ; j, k-1\rangle+|\sigma-i b ; j, k-1\rangle, \quad k \geq 2 .
$$

The relation among boundary states proposed in [14] follows by linear superposition from this equality together with an analogous one involving shifts of the $j$ index and shifts of $\sigma$ by $i / b$.

In section 4, we construct a difference equation that is satisfied by a certain class of correlators in minimal string theory, again by evaluating the ground ring operators' action on the tachyons in the correlator in different ways. The most general correlators can be analyzed along similar lines. This generalizes a similar equation obtained in [15], where a Neumann boundary condition was considered for the matter CFT. Finally we end with a discussion.

\section{Disc correlators with only one boundary insertion}

In this section, we discuss disc correlators with no closed string vertex operators and with only one open string vertex operator inserted on the boundary. There are particular ground ring elements for which $\sigma$ and $(m, n)$ do not change across their insertion points. From our previous discussion, we see that they correspond to the choices $r=2 l+1, s=2 k+1$, where $0 \leq 2 l<q-1$ and $0 \leq 2 k<p-1$. Hence the disc one-point function for such an operator does not naively vanish by any symmetry arguments. In particular, considering the boundary ground ring elements, we see that the choice $k=l=0$ gives the disc partition function with $\sigma$ and $(m, n)$ boundary conditions, which is of course non-vanishing. We now 
show that

$$
\left\langle{ }_{(m, n)}^{\sigma}\left[\mathcal{O}_{2 l+1,2 k+1}\right]_{(m, n)}^{\sigma}\right\rangle=0,
$$

for $k, l \geq 1$, which will be useful later. Also it will follow trivially from our analysis that this statement is true for any such one-point function: except the partition function, all disc amplitudes with only one boundary insertion vanish.

In order to compute the disc correlator for the ground ring, we consider the equation

$$
\left.\frac{\partial^{2}}{\partial \mu_{B}^{2}}\left\langle{ }_{(m, n)}^{\sigma}\left[\mathcal{O}_{2 l+1,2 k+1}\right]_{(m, n)}^{\sigma}\right\rangle\right|_{\mu}=\left\langle{ }_{(m, n)}^{\sigma}\left[T_{1,1}\right]_{(m, n)}^{\sigma}\left[T_{1,1}\right]_{(m, n)}^{\sigma}\left[\mathcal{O}_{2 l+1,2 k+1}\right]_{(m, n)}^{\sigma}\right\rangle,
$$

where $\underset{(m, n)}{\sigma}\left[T_{1,1}\right]_{(m, n)}^{\sigma}=c \underset{(m, n)}{\sigma}\left[e^{b \phi}\right]_{(m, n)}^{\sigma}$ is the boundary Liouville interaction. We have inserted two boundary tachyons, which fixes a part of the conformal killing group of the disc leaving a residual finite part, so the correlator does not vanish trivially. The threepoint function is given by $\mathcal{L}_{2 l+1,2 k+1}$ acting on a product of Liouville and matter three-point functions. The Liouville correlator can be computed using the fact that the ground ring element is a degenerate primary (this approach was originally proposed by Teschner in computing bulk Liouville correlators [16]) and yields a finite number of terms in its OPE with $T_{1,1}$, one of which is $T_{1,1}$ itself. Thus the correlator reduces to a two-point function of $T_{1,1}$ 's which is non-vanishing [10]. One can also calculate it directly using the expression for the boundary three-point function [12].

Another way to do the computation is to consider the equation

$$
\left.\frac{\partial}{\partial \mu}\left\langle{ }_{(m, n)}^{\sigma}\left[\mathcal{O}_{2 l+1,2 k+1}\right]_{(m, n)}^{\sigma}\right\rangle\right|_{\mu_{B}}=-\left\langle\left(c \bar{c} e^{2 b \phi}\right)_{(m, n)}^{\sigma}\left[\mathcal{O}_{2 l+1,2 k+1}\right]_{(m, n)}^{\sigma}\right\rangle,
$$

and compute the bulk-boundary structure constant using expressions given in $[17,18]$. So the Liouville part of the one-point function is non-vanishing and finite.

In order to compute the matter correlator, let us consider the three-point function of the Virasoro primaries

$$
\left\langle{ }^{A}\left[V_{I}^{(\mathrm{M})}\right]^{B}\left[V_{J}^{(\mathrm{M})}\right]^{C}\left[V_{K}^{(\mathrm{M})}\right]^{A}\right\rangle=C_{I J K}^{A B C},
$$

for any rational CFT, where we are interested in the particular case when two of the operators are identity. Alternatively, we could have also considered the bulk-boundary correlator

$$
\left\langle\mathcal{V}_{I}^{(\mathrm{M})}{ }^{A}\left[V_{J}^{(\mathrm{M})}\right]^{A}\right\rangle
$$

where $\mathcal{V}^{(\mathrm{M})}$ is a bulk operator, which is the identity in our case. 
The structure constant (20) can be deduced and essentially depends on the fusion (F) matrix of the RCFT. ${ }^{5}$ In fact, using Lewellen's sewing constraints [19] on the disc, the structure constant (20) has been deduced in [20] for the A-series Virasoro minimal models (the generalization to the $\mathrm{D}$-series has also been done in [21]). Upto freedom of rescaling fields, Runkel has argued that

$$
C_{I J K}^{A B C}=F_{B K}\left[\begin{array}{cc}
A & C \\
I & J
\end{array}\right]
$$

The same conclusion has also been reached by other authors [22,23], who have used different normalizations. In [22], the ratio of the three-point function to the two-point function is given by the fusion matrix

$$
\frac{C_{I J K}^{A B C}}{C_{K K}^{A C}}=F_{B K}\left[\begin{array}{cc}
A & C \\
I & J
\end{array}\right],
$$

while in [23], the authors have normalized the correlator as

$$
\frac{C_{I J K}^{A B C}}{C_{I J K}}=\sqrt{\frac{S_{I 0} S_{J 0}}{S_{K 0} S_{00}}}\left\{\begin{array}{ccc}
I & A & B \\
C & J & K
\end{array}\right\}=\sqrt{\frac{S_{I 0} S_{J 0}}{S_{K 0} S_{00}}} F_{B K}\left[\begin{array}{cc}
A & C \\
I & J
\end{array}\right],
$$

where $S_{I J}$ is the modular matrix, $\{\cdots\}$ is the quantum $6 j$ symbol and $C_{I J K}$ is the structure constant for the bulk three-point function on the sphere. The last equality in the above equation follows from the discussion below.

Using the quantum group approach to studying rational CFTs, it can be shown that the quantum $6 j$ symbols solve the polynomial equations of Moore and Seiberg [24,25]. In fact, the fusion matrix is related to the quantum $6 j$ symbol by the equation $[26,27]$

$$
F_{I J}\left[\begin{array}{ll}
K & L \\
M & N
\end{array}\right]=\left\{\begin{array}{lll}
M & K & I \\
L & N & J
\end{array}\right\} .
$$

The definition of the $6 j$ symbol (see [28] for its explicit form) involves a parameter $q$ of the quantum group $S U_{q}(2) \cdot{ }^{6}$

So for our purposes, from (18) the three-point function is proportional to

$$
C_{(1,1)(1,1)(2 l+1,2 k+1)}^{(m, n)(m, n)(m, n)}=F_{(m, n)(2 l+1,2 k+1)}\left[\begin{array}{cc}
(1,1) & (1,1) \\
(m, n) & (m, n)
\end{array}\right]=0 .
$$

This vanishes for $k, l \geq 1$ trivially as $(1,1) \times(1,1) \rightarrow(2 l+1,2 k+1)$ has vanishing fusion rule, leading to (17). In fact, from (22) it follows that the boundary three-point function

\footnotetext{
${ }^{5}$ The bulk-boundary structure constant (21) depends on the fusion matrix and also on the modular matrix element for the torus with one operator insertion.

${ }^{6}$ Not to be confused with the label $q$ of the $(p, q)$ minimal model.
} 
is non-vanishing only if the three boundary indices $I, J$ and $K$ have non-zero fusion rule. So all boundary one-point functions vanish (except the partition function where the three operators are all the identity) on the disc.

\section{Basic relations among boundary states}

We now use the boundary ground ring to motivate various relations among boundary states, for which we also present some evidence. The general strategy is to consider the disc amplitude with arbitrary bulk vertex operator insertions, and two boundary ground ring insertions compatible with the choice of boundary conditions. Using the fact that any correlator is independent of the position of insertion of a ground ring element, we can contract the two ground ring elements in two ways along the boundary, see figure 1 .

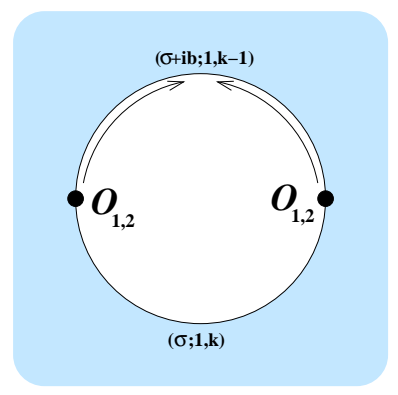

(a)

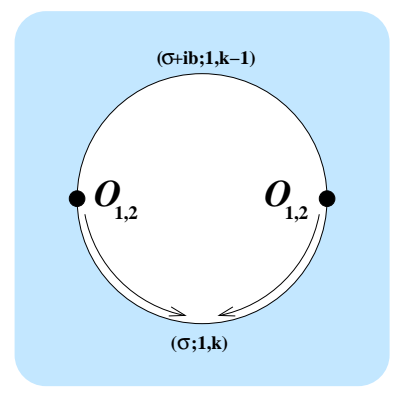

(b)

Figure 1: Utilizing two ways of contracting ground ring operators along the boundary yields an identity on correlation functions.

This gives a relation among correlators with the same bulk insertions but different boundary insertions and different boundary conditions. We shall obtain such relations involving partition functions, and motivate relations among boundary states in the full theory from them.

To begin with, we consider the two-point functions

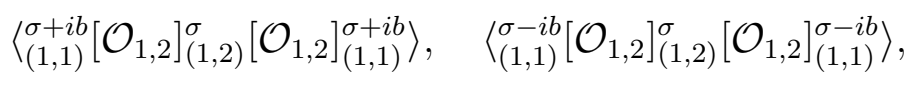

and evaluate them separately. Here on one segment of the disc we have boundary conditions $\{\sigma,(1,2)\}$, while on the other segment we consider both the possibilities $\sigma \pm i b$ allowed by the 
insertion of $\mathcal{O}_{1,2}$, while fixing the matter boundary condition to be $(1,1)$. This construction will motivate a certain relation among correlators, leading us to generalize this analysis later.

Each correlator factorizes into a product of Liouville and matter correlators. We shall neglect all factors that are independent of the boundary conditions, so factors arising from $\mathcal{L}_{1,2}$ will be dropped. We first evaluate

$$
\left\langle\begin{array}{l}
\sigma+i b \\
(1,1)
\end{array}\left[\mathcal{O}_{1,2}\right]_{(1,2)}^{\sigma}\left[\mathcal{O}_{1,2}\right]_{(1,1)}^{\sigma+i b}\right\rangle
$$

by contracting $\mathcal{O}_{1,2} \cdot \mathcal{O}_{1,2}$ along $(\sigma+i b ; 1,1)$. This yields ${ }^{7}$

$$
\left\langle{ }_{(1,1)}^{\sigma+i b}\left[\mathcal{O}_{1,2}\right]_{(1,2)}^{\sigma}\left[\mathcal{O}_{1,2}\right]_{(1,1)}^{\sigma+i b}\right\rangle=\lambda_{L} \lambda_{M} Z(\sigma ; 1,2),
$$

where

$$
\begin{aligned}
& \sigma\left[e^{-b \phi / 2}\right]^{\sigma+i b}\left[e^{-b \phi / 2}\right]^{\sigma} \sim{ }^{\sigma}\left[e^{-b \phi}\right]^{\sigma}+\lambda_{L}{ }^{\sigma}[1]^{\sigma} \\
& { }_{(1,2)}\left[e^{i b X / 2}\right]_{(1,1)}\left[e^{i b X / 2}\right]_{(1,2)} \sim{ }_{(1,2)}\left[e^{i b X}\right]_{(1,2)}+\lambda_{M(1,2)}[1]_{(1,2)} .
\end{aligned}
$$

On the other hand, contracting $\mathcal{O}_{1,2} . \mathcal{O}_{1,2}$ along $(\sigma ; 1,2)$ yields

$$
\left\langle{ }_{(1,1)}^{\sigma+i b}\left[\mathcal{O}_{1,2}\right]_{(1,2)}^{\sigma}\left[\mathcal{O}_{1,2}\right]_{(1,1)}^{\sigma+i b}\right\rangle=\lambda_{L}^{\prime} \lambda_{M}^{\prime} Z(\sigma+i b ; 1,1),
$$

where

$$
\begin{gathered}
{ }^{\sigma+i b}\left[e^{-b \phi / 2}\right]^{\sigma}\left[e^{-b \phi / 2}\right]^{\sigma+i b} \sim{ }^{\sigma+i b}\left[e^{-b \phi}\right]^{\sigma+i b}+\lambda_{L}^{\prime}{ }^{\sigma+i b}[1]^{\sigma+i b} \\
(1,1)\left[e^{i b X / 2}\right]_{(1,2)}\left[e^{i b X / 2}\right]_{(1,1)} \sim{ }_{(1,1)}\left[e^{i b X}\right]_{(1,1)}+\lambda_{M(1,1)}^{\prime}[1]_{(1,1)},
\end{gathered}
$$

leading to the relation

$$
\left\langle{ }_{(1,1)}^{\sigma+i b}\left[\mathcal{O}_{1,2}\right]_{(1,2)}^{\sigma}\left[\mathcal{O}_{1,2}\right]_{(1,1)}^{\sigma+i b}\right\rangle=\lambda_{L} \lambda_{M} Z(\sigma ; 1,2)=\lambda_{L}^{\prime} \lambda_{M}^{\prime} Z(\sigma+i b ; 1,1)
$$

Similarly for the other correlator in (27), we get

$$
\left\langle\begin{array}{l}
\sigma-i b \\
(1,1)
\end{array}\left[\mathcal{O}_{1,2}\right]_{(1,2)}^{\sigma}\left[\mathcal{O}_{1,2}\right]_{(1,1)}^{\sigma-i b}\right\rangle=\widetilde{\lambda}_{L} \lambda_{M} Z(\sigma ; 1,2)=\widetilde{\lambda}_{L}^{\prime} \lambda_{M}^{\prime} Z(\sigma-i b ; 1,1),
$$

\footnotetext{
${ }^{7}$ In all these calculations involving no bulk operator insertions, only the partition function survives the contraction-all the other one-point functions involve the boundary operator $\mathcal{O}_{1,3}$ and vanish using the arguments of the previous section.
} 
where

$$
\begin{aligned}
& \sigma\left[e^{-b \phi / 2}\right]^{\sigma-i b}\left[e^{-b \phi / 2}\right]^{\sigma} \sim{ }^{\sigma}\left[e^{-b \phi}\right]^{\sigma}+\widetilde{\lambda}_{L}{ }^{\sigma}[1]^{\sigma}, \\
& \sigma-i b\left[e^{-b \phi / 2}\right]^{\sigma}\left[e^{-b \phi / 2}\right]^{\sigma-i b} \sim^{\sigma-i b}\left[e^{-b \phi}\right]^{\sigma-i b}+\widetilde{\lambda}_{L}^{\prime}{ }^{\sigma-i b}[1]^{\sigma-i b} .
\end{aligned}
$$

Thus from (33) and (34) we get

$$
Z(\sigma+i b ; 1,1)+Z(\sigma-i b ; 1,1)=\frac{\lambda_{M}}{\lambda_{M}^{\prime}}\left(\frac{\lambda_{L}}{\lambda_{L}^{\prime}}+\frac{\widetilde{\lambda}_{L}}{\widetilde{\lambda}_{L}^{\prime}}\right) Z(\sigma ; 1,2) .
$$

We now proceed to calculate the various coefficients in this relation. From (30), we see that $\lambda_{L}$ can be calculated perturbatively using screening integrals [29-31]. We get

$$
-\lambda_{L}=\mu_{B}^{i} \int_{C_{i}} d x\left\langle{ }^{\sigma}\left[e^{-b \phi / 2}(0)\right]^{\sigma+i b}\left[e^{-b \phi / 2}(1)\right]^{\sigma}\left[e^{Q_{L} \phi}(\infty)\right]^{\sigma}\left[e^{b \phi}(x)\right]\right\rangle,
$$

where

$$
\mu_{B}^{i}= \begin{cases}\sqrt{\mu} \cosh \pi b \sigma, & C_{i} \in(-\infty, 0), \\ \sqrt{\mu} \cosh \pi b(\sigma+i b), & C_{i} \in[0,1), \\ \sqrt{\mu} \cosh \pi b \sigma, & C_{i} \in[1, \infty) .\end{cases}
$$

Now (37) can be evaluated using free field theory, and gives

$$
\lambda_{L}=-\sqrt{\mu} B\left(1+b^{2}, 1+b^{2}\right)\left[\cosh \pi b(\sigma+i b)-\frac{\cosh \pi b \sigma}{\cos \pi b^{2}}\right] .
$$

Similarly we get

$$
\begin{aligned}
& \lambda_{L}^{\prime}=-\sqrt{\mu} B\left(1+b^{2}, 1+b^{2}\right)\left[\cosh \pi b \sigma-\frac{\cosh \pi b(\sigma+i b)}{\cos \pi b^{2}}\right], \\
& \tilde{\lambda}_{L}=-\sqrt{\mu} B\left(1+b^{2}, 1+b^{2}\right)\left[\cosh \pi b(\sigma-i b)-\frac{\cosh \pi b \sigma}{\cos \pi b^{2}}\right], \\
& \widetilde{\lambda}_{L}^{\prime}=-\sqrt{\mu} B\left(1+b^{2}, 1+b^{2}\right)\left[\cosh \pi b \sigma-\frac{\cosh \pi b(\sigma-i b)}{\cos \pi b^{2}}\right] .
\end{aligned}
$$

Straightforward algebra shows that

$$
\frac{\lambda_{L}}{\lambda_{L}^{\prime}}+\frac{\widetilde{\lambda}_{L}}{\widetilde{\lambda}_{L}^{\prime}}=-2 \cos \pi b^{2}
$$

Note that this combination is independent of $\sigma$. For $\lambda_{M}$ and $\lambda_{M}^{\prime}$, using the normalization in (22), we get

$$
\lambda_{M}=F_{(1,1)(1,1)}\left[\begin{array}{ll}
(1,2) & (1,2) \\
(1,2) & (1,2)
\end{array}\right]
$$


and

$$
\lambda_{M}^{\prime}=F_{(1,2)(1,1)}\left[\begin{array}{ll}
(1,1) & (1,1) \\
(1,2) & (1,2)
\end{array}\right] .
$$

In any RCFT, we have the relations [25]

$$
F_{00}\left[\begin{array}{ll}
I & I \\
I & I
\end{array}\right]=\frac{S_{00}}{S_{0 I}}
$$

and

$$
F_{K 0}\left[\begin{array}{ll}
I & I \\
J & J
\end{array}\right]=\frac{S_{0 K}}{S_{0 J}} F_{J 0}\left[\begin{array}{cc}
K & K \\
I & I
\end{array}\right]
$$

leading to

$$
\frac{\lambda_{M}}{\lambda_{M}^{\prime}}=\frac{S_{(1,1)(1,1)}}{S_{(1,1)(1,2)}}
$$

Then (36) gives

$$
Z(\sigma+i b ; 1,1)+Z(\sigma-i b ; 1,1)=-2 \cos \pi b^{2} \frac{S_{(1,1)(1,1)}}{S_{(1,1)(1,2)}} Z(\sigma ; 1,2) .
$$

Using the expression for the modular matrix

$$
S_{(r, s)\left(r^{\prime}, s^{\prime}\right)}=2 \sqrt{\frac{2}{p q}}(-1)^{1+r s^{\prime}+r^{\prime} s} \sin \frac{\pi p r r^{\prime}}{q} \sin \frac{\pi q s s^{\prime}}{p}
$$

we see that

$$
\frac{S_{(1,1)(1,1)}}{S_{(1,1)(1,2)}}=-\frac{1}{2 \cos \pi b^{2}}
$$

Thus (47) leads to

$$
\langle 0 \mid \sigma ; 1,2\rangle=\langle 0 \mid \sigma+i b ; 1,1\rangle+\langle 0 \mid \sigma-i b ; 1,1\rangle .
$$

We have thus arrived at a relation between correlators with different Liouville and matter content, where the shift in matter content is accompanied by an imaginary shift in $\sigma$. We now obtain a generalization of (150) to all disk partition functions.

We consider the correlator

$$
\left\langle\underset{(1, k-1)}{\sigma+i b}\left[\mathcal{O}_{1,2}\right]_{(1, k)}^{\sigma}\left[\mathcal{O}_{1,2}\right]_{(1, k-1)}^{\sigma+i b}\right\rangle
$$

and evaluate it in two ways as mentioned above. We get that

$$
\left\langle\begin{array}{l}
\sigma+i b \\
(1, k-1)
\end{array}\left[\mathcal{O}_{1,2}\right]_{(1, k)}^{\sigma}\left[\mathcal{O}_{1,2}\right]_{(1, k-1)}^{\sigma+i b}\right\rangle=\lambda_{L} \widetilde{\lambda}_{M} Z(\sigma ; 1, k)=\lambda_{L}^{\prime} \widetilde{\lambda}_{M}^{\prime} Z(\sigma+i b ; 1, k-1),
$$


where

$$
\widetilde{\lambda}_{M}=F_{(1, k-1)(1,1)}\left[\begin{array}{ll}
(1, k) & (1, k) \\
(1,2) & (1,2)
\end{array}\right]
$$

and

$$
\widetilde{\lambda}_{M}^{\prime}=F_{(1, k)(1,1)}\left[\begin{array}{cc}
(1, k-1) & (1, k-1) \\
(1,2) & (1,2)
\end{array}\right] .
$$

Thus using (45), we get

$$
\frac{\widetilde{\lambda}_{M}}{\widetilde{\lambda}_{M}^{\prime}}=\frac{S_{(1,1)(1, k-1)}}{S_{(1,1)(1, k)}}
$$

We also evaluate the correlator with two boundary $\mathcal{O}_{1,2}$ insertions, $(\sigma, \sigma-i b)$ boundary conditions and same matter boundary conditions, to get

$$
\left\langle\begin{array}{l}
\sigma-i b \\
(1, k-1)
\end{array}\left[\mathcal{O}_{1,2}\right]_{(1, k)}^{\sigma}\left[\mathcal{O}_{1,2}\right]_{(1, k-1)}^{\sigma-i b}\right\rangle=\widetilde{\lambda}_{L} \widetilde{\lambda}_{M} Z(\sigma ; 1, k)=\widetilde{\lambda}_{L}^{\prime} \widetilde{\lambda}_{M}^{\prime} Z(\sigma-i b ; 1, k-1) .
$$

Thus

$$
Z(\sigma+i b ; 1, k-1)+Z(\sigma-i b ; 1, k-1)=-2 \cos \pi b^{2} \frac{S_{(1,1)(k-1)}}{S_{(1,1)(1, k)}} Z(\sigma ; 1, k) .
$$

Unlike (501), the right-hand side of (57) depends on the boundary condition in the matter sector. We would like to deduce an equation free from this constraint, and so using the relation in the matter sector

$$
Z(m, n)=\frac{S_{(1,1)(m, n)}}{S_{(1,1)\left(m^{\prime}, n^{\prime}\right)}} Z\left(m^{\prime}, n^{\prime}\right)
$$

which follows from [9]

$$
Z(m, n)=\frac{S_{(1,1)(m, n)}}{\sqrt{S_{(1,1)(1,1)}}}
$$

we rewrite (57) as

$$
\begin{aligned}
Z(\sigma+i b ; 1, k-1)+Z(\sigma-i b ; 1, k-1) & =-2 \cos \pi b^{2} \frac{S_{(1,1)(1, k-1)}}{S_{(1,1)(1, k)}} Z(\sigma ; 1, k) \\
& =-2 \cos \pi b^{2} \frac{S_{(1,1)(1, k-1)}}{S_{(1,1)(1, k-2)}} Z(\sigma ; 1, k-2) .
\end{aligned}
$$

From (60), it follows that

$$
\begin{aligned}
Z(\sigma ; 1, k)+Z(\sigma ; 1, k-2)= & -\frac{1}{2 \cos \pi b^{2}} \frac{S_{(1,1)(1, k)}+S_{(1,1)(k-2)}}{S_{(1,1)(k-1)}} \\
& \times[Z(\sigma+i b ; 1, k-1)+Z(\sigma-i b ; 1, k-1)] .
\end{aligned}
$$


On using (48), we simplify (61) to get

$$
\langle 0 \mid \sigma ; 1, k\rangle+\langle 0 \mid \sigma ; 1, k-2\rangle=\langle 0 \mid \sigma+i b ; 1, k-1\rangle+\langle 0 \mid \sigma-i b ; 1, k-1\rangle,
$$

for $k \geq 2$ (in fact (50) is the particular case with $k=2$ ). Note that this equation relates correlators with coefficients independent of boundary conditions, and involves only $(1, k),(1, k-1),(1, k-2)$ Cardy boundary states and $(\sigma, \sigma+i b, \sigma-i b)$ FZZT boundary states. Applied recursively, it gives a set of relations among correlators.

Now (62) by itself is a relation among disc partition functions, which we want to elevate to a relation among boundary states in the full theory, modulo BRST exact states. We propose the relation among boundary states

$$
|\sigma ; 1, k\rangle+|\sigma ; 1, k-2\rangle=|\sigma+i b ; 1, k-1\rangle+|\sigma-i b ; 1, k-1\rangle, \quad k \geq 2 .
$$

We have established this equality at the level of the disc partition function above. To provide more evidence, we calculate the disc one-point function with one bulk vertex operator insertion and check the equality. This analysis is very similar to the one in [14], to which we shall soon return.

The boundary state $|\sigma ; 1, s\rangle$ is given by

$$
|\sigma ; 1, s\rangle=\sum_{m, n} \int_{0}^{\infty} \frac{d p}{\pi} \cos (2 \pi \sigma p) U^{*}(p) \frac{S_{(1, s)(m, n)}}{\sqrt{S_{(1,1)(m, n)}}}|p \gg| m, n \gg,
$$

where $\mid p \gg$ and $\mid m, n \gg$ are Liouville and matter Ishibashi states $[32,33]$ respectively, and $[10]$

$$
U(p)=\left(\pi \mu \gamma\left(b^{2}\right)\right)^{-i p / b} \frac{\Gamma(1+2 i p b) \Gamma\left(1+\frac{2 i p}{b}\right)}{i p} .
$$

Considering the bulk tachyon vertex operator

$$
\mathcal{T}_{r, s}=c \bar{c} e^{2 i \alpha_{r, s} X} e^{2 \beta_{r, s} \phi}
$$

and dropping all factors independent of the boundary conditions, we get

$$
\begin{aligned}
\left\langle\mathcal{T}_{r, s} \mid \sigma ; 1, k\right\rangle & +\left\langle\mathcal{T}_{r, s} \mid \sigma ; 1, k-2\right\rangle=\left\langle\mathcal{T}_{r, s} \mid \sigma+i b ; 1, k-1\right\rangle+\left\langle\mathcal{T}_{r, s} \mid \sigma-i b ; 1, k-1\right\rangle \\
& =(-1)^{s+r k} \cosh \frac{\pi \sigma(p r-q s)}{\sqrt{p q}} \sin \frac{\pi r p}{q}\left[\sin \frac{\pi s k q}{p}+\sin \frac{\pi s(k-2) q}{p}\right]
\end{aligned}
$$

We can similarly verify the equality with a bulk ground ring insertion, and the insertion of a bulk physical operator at negative ghost number - the answer goes through with 
appropriate changes in the range of $s$ [14]. Since these are all the physical operators in the theory, the equality (67) provides evidence for (63).

From (63) it follows that a boundary state with Liouville and matter content $(\sigma ; 1, k)$ is given by a linear combination of boundary states with matter content $(1, k-1)$ and $(1, k-2)$ with $\sigma$ appropriately shifted. So using this relation recursively, we can express a boundary state with content $(\sigma ; 1, k)$ as a sum of boundary states with different $\sigma$ but trivial matter content - the $(1,1)$ state. In fact for $k=2$, we already have the expression

$$
|\sigma ; 1,2\rangle=|\sigma+i b ; 1,1\rangle+|\sigma-i b ; 1,1\rangle
$$

For $k=3$, (63) and (68) gives

$$
|\sigma ; 1,3\rangle=|\sigma+2 i b ; 1,1\rangle+|\sigma ; 1,1\rangle+|\sigma-2 i b ; 1,1\rangle
$$

while for $k=4$, (63), (68) and (69) gives

$$
|\sigma ; 1,4\rangle=|\sigma+3 i b ; 1,1\rangle+|\sigma+i b ; 1,1\rangle+|\sigma-i b ; 1,1\rangle+|\sigma-3 i b ; 1,1\rangle,
$$

and so on. In general, we have

$$
|\sigma ; 1, k\rangle=\sum_{m}|\sigma+i m b ; 1,1\rangle
$$

where

$$
m=-(k-1),-(k-1)+2, \ldots, k-1 .
$$

It is easy to see that (71) solves (63) because

$$
\begin{array}{r}
|\sigma ; 1, k\rangle+|\sigma ; 1, k-2\rangle=|\sigma+i b ; 1, k-1\rangle+|\sigma-i b ; 1, k-1\rangle \\
=|\sigma-i b(k-1) ; 1,1\rangle+|\sigma+i b(k-1) ; 1,1\rangle+2 \sum_{t}|\sigma+i b t ; 1,1\rangle,
\end{array}
$$

where

$$
t=-(k-3),-(k-3)+2, \ldots, k-3 .
$$

Replacing $\mathcal{O}_{1,2}$ with $\mathcal{O}_{2,1}$ in the arguments above, gives the other relation

$$
|\sigma ; l, 1\rangle+|\sigma ; l-2,1\rangle=\left|\sigma+\frac{i}{b} ; l-1,1\right\rangle+\left|\sigma-\frac{i}{b} ; l-1,1\right\rangle, \quad l \geq 2,
$$

leading to

$$
|\sigma ; l, 1\rangle=\sum_{n}\left|\sigma+\frac{i n}{b} ; 1,1\right\rangle,
$$


where

$$
n=-(l-1),-(l-1)+2, \ldots, l-1 \text {. }
$$

Using the independence of shifts by $b$ and $1 / b$, (711) and (76) together give

$$
|\sigma ; l, k\rangle=\sum_{m, n}\left|\sigma+i m b+\frac{i n}{b} ; 1,1\right\rangle,
$$

where $m$ and $n$ are given by (72) and (77). Now (78) relates a boundary state with arbitrary Liouville and matter content to a sum of boundary states with Liouville content changed by imaginary shifts in $\sigma$, and trivial matter content. This is precisely the relation obtained by Seiberg and Shih in [14], which follows as a consequence of (63) and (75).

We see that the relation (78) follows naturally using the boundary ground ring. Essentially in (78) the shift in $\sigma$ is given by multiples of $b$ and $1 / b$, which is the same as the change $\sigma_{L}-\sigma_{R}$ (or $\sigma_{L}+\sigma_{R}$ ) across insertions of boundary ground ring elements. In fact, across the insertion of the ring element $\mathcal{O}_{l, k}$, the change in $\sigma$ is given by $\sigma_{L}-\sigma_{R}$ (or $\left.\sigma_{L}+\sigma_{R}\right)=i m b+i n / b[10]$, where $m$ and $n$ are exactly as given in (72) and (77). Thus such a shift relation has a natural interpretation as shifts due to the degenerate structure of the boundary ground ring.

\section{A difference equation for boundary correlators}

The boundary ground ring can also be used to constrain correlation functions involving boundary vertex operator insertions [13]. For simplicity, we restrict the discussion to disc correlators which contain only boundary vertex operators. An equation satisfied by such correlators has been obtained in [15], where a Neumann boundary condition was imposed for the matter CFT, which we generalize to Liouville theory coupled to minimal models. ${ }^{8}$ We consider certain correlators and show that they satisfy a difference equation using the fact that the tachyons form a module under the action of the ground ring $[13,34]$ in both open and closed string theory.

The two basic ground ring elements are

$$
\begin{aligned}
& \underset{(m, n)}{\sigma}\left[\mathcal{O}_{1,2}\right]_{(m, n \pm 1)}^{\sigma \pm i b}=\left(b c-\frac{1}{b} \partial(\phi+i X)\right){ }^{\sigma}\left[e^{-b \phi / 2}\right]_{(m, n)}^{\sigma \pm i b}\left[e^{i b X / 2}\right]_{(m, n \pm 1)}, \\
& \underset{(m, n)}{\sigma}\left[\mathcal{O}_{2,1}\right]_{(m \pm 1, n)}^{\sigma \pm i / b}=(b c-b \partial(\phi-i X))^{\sigma}\left[e^{-\phi / 2 b}\right]_{(m, n)}^{\sigma \pm i / b}\left[e^{-i X / 2 b}\right]_{(m \pm 1, n)} .
\end{aligned}
$$

${ }^{8}$ Neumann boundary conditions are not among the Cardy boundary conditions. 
First let us consider the theory with $\mu_{B}=0$. Among the boundary tachyons (12), the ones given by $T_{r, 1}(1 \leq r<q)$ and $T_{q-1, s}(1 \leq s<p-p / q)$ form submodules of the tachyon module (this follows exactly as discussed in [34] for the closed string tachyons). The action of the ground ring on these tachyons is given by

$$
\begin{gathered}
(m, n) \\
{\left[\mathcal{O}_{1,2}\right]_{(m, n \pm 1)}\left[T_{r, 1}\right]_{\left(m^{\prime}, n \pm 1\right)}=0} \\
{ }_{(m, n)}\left[\mathcal{O}_{1,2}\right]_{(m, n \pm 1)}\left[T_{q-1, s}\right]_{\left(m, n^{\prime}\right)}=F_{(m, n \pm 1)(q-1, s-1)}\left[\begin{array}{cc}
(m, n) & \left(m, n^{\prime}\right) \\
(1,2) & (q-1, s)
\end{array}\right]{ }_{(m, n)}\left[T_{q-1, s-1}\right]_{\left(m, n^{\prime}\right)} \cdot(
\end{gathered}
$$

Obviously, in the first equation, $\left(m^{\prime}, n \pm 1\right)$ must be in the fusion rule of $(m, n \pm 1) \times(r, 1)$, while in the second equation $\left(m, n^{\prime}\right)$ must be in the fusion rule of $(m, n \pm 1) \times(q-1, s)$ as well as $(m, n) \times(q-1, s-1)$. Analogous statements hold for the equations that follow. Also

$$
\begin{gathered}
(m, n)\left[\mathcal{O}_{2,1}\right]_{(m \pm 1, n)}\left[T_{q-1, s}\right]_{\left(m \pm 1, n^{\prime}\right)}=0 \\
{ }_{(m, n)}\left[\mathcal{O}_{2,1}\right]_{(m \pm 1, n)}\left[T_{r, 1}\right]_{\left(m^{\prime}, n\right)}=F_{(m \pm 1, n)(r+1,1)}\left[\begin{array}{cc}
(m, n) & \left(m^{\prime}, n\right) \\
(2,1) & (r, 1)
\end{array}\right]{ }_{(m, n)}\left[T_{r+1,1}\right]_{\left(m^{\prime}, n\right)} .
\end{gathered}
$$

As before, we have dropped factors independent of boundary conditions - they can be absorbed in the definition of the tachyons, or by suitably rescaling the fusion matrices. The coefficients in these equations are boundary three-point functions and so are given by the fusion matrices. Note that under the interchange of the boundary tachyon and $\mathcal{O}_{1,2}$ (or $\left.\mathcal{O}_{2,1}\right)$ one picks up a sign [13]. For example,

$$
{ }_{(m, n)}\left[T_{r, 1}\right]_{\left(m^{\prime}, n\right)}\left[\mathcal{O}_{2,1}\right]_{\left(m^{\prime} \pm 1, n\right)}=-F_{\left(m^{\prime}, n\right)(r+1,1)}\left[\begin{array}{cc}
(m, n) & \left(m^{\prime} \pm 1, n\right) \\
(r, 1) & (2,1)
\end{array}\right]{ }_{(m, n)}\left[T_{r+1,1}\right]_{\left(m^{\prime} \pm 1, n\right)} .
$$

For the correlators we consider, we need the action of the ground ring on the tachyon module in the presence of ordered integrated boundary vertex operators. We shall consider correlators with a certain ordering, namely the ones which are independent of the position of insertion of the ground ring elements. ${ }^{9}$ In particular, we need to know if the first equation in (80) gets modified. For the correlators we shall consider, we need to know if $\mathcal{O}_{1,2} \cdot T_{r, 1} \cdot \int T_{r^{\prime}, 1}$ is non-vanishing. Just by adding the Liouville momenta, and by minimal model fusion rules, we see that (schematically)

$$
\mathcal{O}_{1,2} \cdot T_{r, 1} \cdot \int T_{r^{\prime}, 1} \sim T_{r+r^{\prime}-1,2}
$$

\footnotetext{
${ }^{9}$ This is not true for the others, because of non-vanishing contributions from the boundary of moduli space $[13]$.
} 
for $2 \leq r+r^{\prime} \leq q$. Explicitly, we get that

$$
\begin{aligned}
& { }_{(m, n)}\left[\mathcal{O}_{1,2}\right]_{(m, n \pm 1)}\left[T_{r, 1}\right]_{\left(m^{\prime}, n \pm 1\right)} \int\left[T_{r^{\prime}, 1}\right]_{\left(m^{\prime \prime}, n \pm 1\right)} \\
& =F_{(m, n \pm 1)(r, 2)}\left[\begin{array}{cc}
(m, n) & \left(m^{\prime}, n \pm 1\right) \\
(1,2) & (r, 1)
\end{array}\right] F_{\left(m^{\prime}, n \pm 1\right)\left(r+r^{\prime}-1,2\right)}\left[\begin{array}{cc}
(m, n) & \left(m^{\prime \prime}, n \pm 1\right) \\
(r, 2) & \left(r^{\prime}, 1\right)
\end{array}\right] \\
& \times_{(m, n)}\left[T_{r+r^{\prime}-1,2}\right]_{\left(m^{\prime \prime}, n \pm 1\right) .}
\end{aligned}
$$

The coefficient in (84) is a product of fusion matrices because it is a four-point boundary correlator which equals the sum over intermediate channels of products of boundary threepoint functions consistent with the fusion rules (in this case there is only one channel $(1,2) \times(r, 1) \rightarrow(r, 2))$.

Similarly, because of the ordered nature of the integrated vertex operator, we have another relation (though we shall not need it, we list it for the sake of completeness)

$$
\begin{aligned}
& \int_{(m, n)}\left[T_{r, 1}\right]_{\left(m^{\prime}, n\right)}\left[\mathcal{O}_{1,2}\right]_{\left(m^{\prime}, n \pm 1\right)}\left[T_{r^{\prime}, 1}\right]_{\left(m^{\prime \prime}, n \pm 1\right)} \\
& =F_{\left(m^{\prime}, n\right)(r, 2)}\left[\begin{array}{cc}
(m, n) & \left(m^{\prime}, n \pm 1\right) \\
(r, 1) & (1,2)
\end{array}\right] F_{\left(m^{\prime}, n \pm 1\right)\left(r+r^{\prime}-1,2\right)}\left[\begin{array}{cc}
(m, n) & \left(m^{\prime \prime}, n \pm 1\right) \\
(r, 2) & \left(r^{\prime}, 1\right)
\end{array}\right] \\
& \times_{(m, n)}\left[T_{r+r^{\prime}-1,2}\right]_{\left(m^{\prime \prime}, n \pm 1\right)} .
\end{aligned}
$$

Once again, the coefficient is given by a product of fusion matrices. ${ }^{10}$ It should be noted that the coefficients in (84) and (85) involve squares of fusion matrices only when the matter part of the integrated vertex operator has a non-trivial OPE with the other two vertex operators. For example, consider the case when the integrated vertex operator is the boundary cosmological constant, which contains the identity in the matter part. Then in the matter sector there are no singularites that arise due to contact terms of the other two operators with the identity, and then the coefficients are different. In fact they are then given by matter three-point functions, and so they are linear (and not quadratic) in the fusion matrices.

Now consider the case when $\mu_{B}$ is turned on, i.e. in the presence of FZZT branes. We shall consider the equations involving $\mathcal{O}_{1,2}$, the results for the equations involving $\mathcal{O}_{2,1}$ follow by replacing $b \rightarrow 1 / b, \mu_{B} \rightarrow \widetilde{\mu}_{B}$, and suitably interchanging the Kac table indices. We want to see how (80) and (84) are modified in the presence of FZZT branes. The

\footnotetext{
${ }^{10}$ For $c_{M}=1$, the corresponding coefficients involve trigonometric functions and were derived in [13] for open string theory. For closed string theory, the coefficient is given by the Virasoro-Shapiro four-point function and was derived in $[35,36]$.
} 
Liouville part of the answer can be worked out along the lines of the previous section as it involves inserting one boundary cosmological constant, and the minimal model part gives the fusion matrix. The first equation in (80) gets modified to

$$
\begin{aligned}
& \underset{(m, n)}{\sigma}\left[\mathcal{O}_{1,2}\right]_{(m, n \pm 1)}^{\sigma \pm i b}\left[T_{r, 1}\right]_{\left(m^{\prime}, n \pm 1\right)}^{\sigma^{\prime}}=-\sqrt{\mu} \frac{r-b^{2}}{b^{2} \sin 2 \pi b^{2}} B\left(1+b^{2}, b^{2}-r\right) \\
& \times\left(\sin 2 \pi b^{2} \cosh \pi b \sigma-\sin \pi b^{2}\left\{\cosh \pi b(\sigma \pm i b)-(-1)^{r} \cosh \pi b \sigma^{\prime}\right\}\right) \\
& \times F_{(m, n \pm 1)(r, 2)}\left[\begin{array}{cc}
(m, n) & \left(m^{\prime}, n \pm 1\right) \\
(1,2) & (r, 1)
\end{array}\right] \underset{(m, n)}{\sigma}\left[T_{r, 2}\right]_{\left(m^{\prime}, n \pm 1\right)}^{\sigma^{\prime}},
\end{aligned}
$$

while the second equation in (80) gets modified to

$$
\begin{aligned}
& \underset{(m, n)}{\sigma}\left[\mathcal{O}_{1,2}\right]_{(m, n \pm 1)}^{\sigma \pm i b}\left[T_{q-1, s}\right]_{\left(m, n^{\prime}\right)}^{\sigma^{\prime}} \\
& =\frac{1-q+s b^{2}}{b^{2}} F_{(m, n \pm 1)(q-1, s-1)}\left[\begin{array}{cc}
(m, n) & \left(m, n^{\prime}\right) \\
(1,2) & (q-1, s)
\end{array}\right]{ }_{(m, n)}^{\sigma}\left[T_{q-1, s-1}\right]_{\left(m, n^{\prime}\right)}^{\sigma^{\prime}} \\
& +\sqrt{\mu} \frac{1-q+s b^{2}}{b^{2} \sin (1+s) \pi b^{2}} B\left(1+b^{2}, 1-q+s b^{2}\right) \\
& \times\left(\sin (1+s) \pi b^{2} \cosh \pi b \sigma-\sin \pi s b^{2} \cosh \pi b(\sigma \pm i b)-(-1)^{q} \sin \pi b^{2} \cosh \pi b \sigma^{\prime}\right) \\
& \times F_{(m, n \pm 1)(q-1, s+1)}\left[\begin{array}{cc}
(m, n) & \left(m, n^{\prime}\right) \\
(1,2) & (q-1, s)
\end{array}\right] \underset{(m, n)}{\sigma}\left[T_{q-1, s+1}\right]_{\left(m, n^{\prime}\right)}^{\sigma^{\prime}} .
\end{aligned}
$$

We have included the numerical factors that arise from the ghost, $L_{-1}^{M}$ and $L_{-1}^{L}$ prefactors in (79). This also leads to a multiplicative factor of

$$
\frac{\left(r+r^{\prime}\right)\left(1+b^{2}\right)-r r^{\prime}-b^{2} Q_{L}^{2}}{b^{4}}
$$

in (84).

Finally, we argue that (84) does not receive any corrections. At $O(1), T_{r+r^{\prime}-1,2}$ emerges, as we saw before. At $O\left(\mu_{B}\right)$, by comparing Liouville momentum, $T_{r+r^{\prime}-1,4}$ should emerge. Similarly, at $O\left(\mu_{B}^{2}\right), T_{r+r^{\prime}-1,6}$ should emerge, and so on. However, from the left-hand side of (84), using the minimal model fusion rules, we see that only tachyons of the form $T_{k, 2}$ can emerge. Hence, all terms except the $O(1)$ term vanish. It is also easy to see the vanishing in terms of the fusion matrix. For example, at $O\left(\mu_{B}\right)$, this is due to the factor of

$$
F_{\left(m^{\prime}, n \pm 1\right)\left(r+r^{\prime}-1,4\right)}\left[\begin{array}{cc}
(m, n) & \left(m^{\prime \prime}, n+1\right) \\
(r, 2) & \left(r^{\prime}, 1\right)
\end{array}\right]=0
$$

which enters the matter four-point function. 
We now deduce a difference equation satisfied by boundary correlators. Generic correlators of the form

$$
\left\langle\begin{array}{l}
\sigma_{1} \\
\left(m_{1}, n_{1}\right)
\end{array}\left[T_{r_{1}, s_{1}}\right]_{\left(m_{2}, n_{2}\right)}^{\sigma_{2}}\left[T_{r_{2}, s_{2}}\right]_{\left(m_{3}, n_{3}\right)}^{\sigma_{3}} \cdots{ }_{\left(m_{k}, n_{k}\right)}^{\sigma_{k}}\left[T_{r_{k}, s_{k}}\right]_{\left(m_{1}, n_{1}\right)}^{\sigma_{1}}\right\rangle
$$

involving only boundary tachyons are cumbersome to manipulate. So we consider a subclass of the most general correlators which are tractable. The arguments we present go through for the general case as well. We consider the correlator

$$
\left\langle\underset{(m, n)}{\sigma}\left[T_{q-1, s}\right]_{\left(m, n^{\prime}\right)}^{\sigma^{\prime}}\left[T_{r, 1}\right]_{\left(m^{\prime}, n^{\prime}\right)}^{\sigma^{\prime \prime}}\left[T_{r^{\prime}, 1}\right]_{\left(m^{\prime \prime}, n^{\prime}\right)}^{\sigma^{\prime \prime \prime}} \cdots\right\rangle
$$

where we have not distinguished between integrated and unintegrated vertex operators for brevity - it should be kept in mind that three of the vertex operators are not integrated while the rest are integrated over in a specific manner as we now discuss. The first three tachyons have been chosen to be of this form so that we can use the ring relations acting on the tachyon module given by (84), (86) and (87), while the remaining tachyons are arbitrary. The tachyons $T_{q-1, s}$ and $T_{r, 1}$ are at 0 and 1 respectively, while the last tachyon in (91) is at $\infty$. Also the integrated tachyons in (91) are ordered along the boundary and not integrated over the whole boundary, in particular, $T_{r^{\prime}, 1}$ is an ordered integrated vertex operator. Thus this correlator gives a particular channel of the scattering amplitude.

Consider the correlator

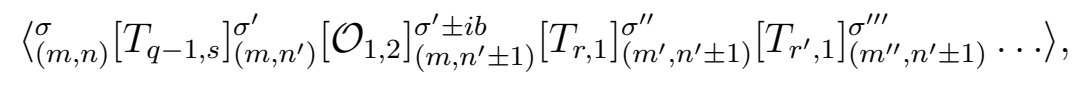

where the various tachyons are inserted on the worldsheet as mentioned above. For this particular choice of insertions, this correlator is independent of the insertion point of $\mathcal{O}_{1,2}$ [13]. We evaluate this correlator in two ways: by allowing $T_{q-1, s} \leftarrow \mathcal{O}_{1,2}$, and $\mathcal{O}_{1,2} \rightarrow T_{r, 1} \int T_{r^{\prime}, 1}$, and equate the answers, on using (84), (86) and (87). This gives us the following relation among correlators

$$
\begin{aligned}
& \alpha \widetilde{M}_{1}\left\langle{ }_{(m, n)}^{\sigma}\left[T_{q-1, s}\right]_{\left(m, n^{\prime}\right)}^{\sigma^{\prime}}\left[T_{r+r^{\prime}-1,2}\right]_{\left(m^{\prime \prime}, n^{\prime} \pm 1\right)}^{\sigma^{\prime \prime \prime}} \cdots\right\rangle \\
& -\sqrt{\mu} \beta f\left(\sigma, \sigma^{\prime}\right) \widetilde{M}_{2}\left\langle{ }_{(m, n)}^{\sigma}\left[T_{q-1, s}\right]_{\left(m, n^{\prime}\right)}^{\sigma^{\prime}}\left[T_{r, 2}\right]_{\left(m^{\prime}, n^{\prime} \pm 1\right)}^{\sigma^{\prime \prime}}\left[T_{r^{\prime}, 1}\right]_{\left(m^{\prime \prime}, n^{\prime} \pm 1\right)}^{\sigma^{\prime \prime \prime}} \ldots\right\rangle \\
& =\gamma \widetilde{M}_{3}\left\langle{ }_{(m, n)}^{\sigma}\left[T_{q-1, s-1}\right]_{\left(m, n^{\prime} \pm 1\right)}^{\sigma^{\prime} \pm i b}\left[T_{r, 1}\right]_{\left(m^{\prime}, n^{\prime} \pm 1\right)}^{\sigma^{\prime \prime}}\left[T_{r^{\prime}, 1}\right]_{\left(m^{\prime \prime}, n^{\prime} \pm 1\right)}^{\sigma^{\prime \prime \prime}} \cdots\right\rangle \\
& -\sqrt{\mu} \tau g\left(\sigma, \sigma^{\prime}\right) \widetilde{M}_{4}\left\langle\left\langle_{(m, n)}^{\sigma}\left[T_{q-1, s+1}\right]_{\left(m, n^{\prime} \pm 1\right)}^{\sigma^{\prime} \pm i b}\left[T_{r, 1}\right]_{\left(m^{\prime}, n^{\prime} \pm 1\right)}^{\sigma^{\prime \prime}}\left[T_{r^{\prime}, 1}\right]_{\left(m^{\prime \prime}, n^{\prime} \pm 1\right)}^{\sigma^{\prime \prime \prime}} \ldots\right\rangle,\right.
\end{aligned}
$$


where

$$
\begin{aligned}
& \alpha=\frac{\left(r+r^{\prime}\right)\left(1+b^{2}\right)-r r^{\prime}-b^{2} Q_{L}^{2}}{b^{4}}, \quad \beta=\frac{r-b^{2}}{b^{2} \sin 2 \pi b^{2}} B\left(1+b^{2}, b^{2}-r\right), \\
& \gamma=\frac{q-1-s b^{2}}{b^{2}}, \quad \tau=-\frac{\gamma}{\sin (1+s) \pi b^{2}} B\left(1+b^{2}, 1-q+s b^{2}\right), \\
& f\left(\sigma, \sigma^{\prime}\right)=\sin 2 \pi b^{2} \cosh \pi b \sigma^{\prime}-\sin \pi b^{2}\left\{\cosh \pi b\left(\sigma^{\prime} \pm i b\right)-(-1)^{r} \cosh \pi b \sigma^{\prime \prime}\right\}, \\
& g\left(\sigma, \sigma^{\prime}\right)=\sin (1+s) \pi b^{2} \cosh \pi b\left(\sigma^{\prime} \pm i b\right)-\sin \pi s b^{2} \cosh \pi b \sigma^{\prime}-(-1)^{q} \sin \pi b^{2} \cosh \pi b \sigma, \\
& \widetilde{M}_{1}=F_{\left(m, n^{\prime} \pm 1\right)(r, 2)}\left[\begin{array}{cc}
\left(m, n^{\prime}\right) & \left(m^{\prime}, n^{\prime} \pm 1\right) \\
(1,2) & (r, 1)
\end{array}\right] F_{\left(m^{\prime}, n^{\prime} \pm 1\right)\left(r+r^{\prime}-1,2\right)}\left[\begin{array}{cc}
\left(m, n^{\prime}\right) & \left(m^{\prime \prime}, n^{\prime} \pm 1\right) \\
(r, 2) & \left(r^{\prime}, 1\right)
\end{array}\right], \\
& \widetilde{M}_{2}=F_{\left(m, n^{\prime} \pm 1\right)(r, 2)}\left[\begin{array}{cc}
\left(m, n^{\prime}\right) & \left(m^{\prime}, n^{\prime} \pm 1\right) \\
(1,2) & (r, 1)
\end{array}\right], \\
& \widetilde{M}_{3}=F_{\left(m, n^{\prime}\right)(q-1, s-1)}\left[\begin{array}{cc}
(m, n) & \left(m, n^{\prime} \pm 1\right) \\
(q-1, s) & (1,2)
\end{array}\right], \\
& \widetilde{M}_{4}=F_{\left(m, n^{\prime}\right)(q-1, s+1)}\left[\begin{array}{cc}
(m, n) & \left(m, n^{\prime} \pm 1\right) \\
(q-1, s) & (1,2)
\end{array}\right] .
\end{aligned}
$$

We want to write down an equation satisfied by (91) starting from (93). In (93) the first term is a correlator with one less tachyon than the others. Also from (94) we see that all the other terms depend on both $\sigma^{\prime} \pm i b$, while the first term in (93) does not. So we take the difference between the two equations (93) involving $\sigma^{\prime}+i b$ and $\sigma^{\prime}-i b$ and thus the first terms drops out. As we now demonstrate, this gives a formal equation satisfied by the correlator (91).

In order to write down the equation, we use the identity in the Liouville sector [15]

$$
\left\langle{ }^{\sigma}[T]^{\sigma^{\prime} \pm i b}[T]^{\sigma^{\prime \prime}}[T] \ldots\right\rangle=e^{ \pm b \partial_{\sigma^{\prime}}}\left\langle{ }^{\sigma}[T]^{\sigma^{\prime}}[T]^{\sigma^{\prime \prime}}[T] \ldots\right\rangle
$$

We need an analogous relation in the matter sector, were the variables are discrete as they lie in the Kac table, and so we define a formal differentiation to shift the indices. As we shall see, this definition is natural given the fusion rule constraint. For the cases we need, we define

$$
\begin{aligned}
& \left\langle{ }_{(m, n)}\left[T_{q-1, s}\right]_{\left(m, n^{\prime}\right)}\left[T_{r, 2}\right]_{\left(m^{\prime}, n^{\prime} \pm 1\right)}\left[T_{r^{\prime}, 1}\right]_{\left(m^{\prime \prime}, n^{\prime} \pm 1\right)} \ldots\right\rangle \\
& =e^{-i \frac{b}{2} \partial_{r, 1}}\left\langle_{(m, n)}\left[T_{q-1, s}\right]_{\left(m, n^{\prime}\right)}\left[T_{r, 1}\right]_{\left(m^{\prime}, n^{\prime}\right)}\left[T_{r^{\prime}, 1}\right]_{\left(m^{\prime \prime}, n^{\prime}\right)} \ldots\right\rangle .
\end{aligned}
$$

The exponential factor adds momentum $b / 2$ to $T_{r, 1}$, and since $\alpha_{r, 1}+b / 2=\alpha_{r, 2}$, we get $T_{r, 2}$. Also the fusion rules between $\left(m, n^{\prime}\right)$ and $(r, 2)$ automatically change the remaining 
index at that insertion point from $\left(m^{\prime}, n^{\prime}\right)$ to $\left(m^{\prime}, n^{\prime} \pm 1\right)$, which changes the index from $\left(m^{\prime \prime}, n^{\prime}\right)$ to $\left(m^{\prime \prime}, n^{\prime} \pm 1\right)$ across $T_{r^{\prime}, 1}$, and so on. Similarly it follows that

$$
\begin{aligned}
& \left\langle{ }_{(m, n)}\left[T_{q-1, s+1}\right]_{\left(m, n^{\prime} \pm 1\right)}\left[T_{r, 1}\right]_{\left(m^{\prime}, n^{\prime} \pm 1\right)}\left[T_{r^{\prime}, 1}\right]_{\left(m^{\prime \prime}, n^{\prime} \pm 1\right)} \ldots\right\rangle \\
& =e^{-i \frac{b}{2} \partial_{q-1, s}}\left\langle_{(m, n)}\left[T_{q-1, s}\right]_{\left(m, n^{\prime}\right)}\left[T_{r, 1}\right]_{\left(m^{\prime}, n^{\prime}\right)}\left[T_{r^{\prime}, 1}\right]_{\left(m^{\prime \prime}, n^{\prime}\right)} \cdots\right\rangle
\end{aligned}
$$

and

$$
\begin{aligned}
& \left\langle{ }_{(m, n)}\left[T_{q-1, s-1}\right]_{\left(m, n^{\prime} \pm 1\right)}\left[T_{r, 1}\right]_{\left(m^{\prime}, n^{\prime} \pm 1\right)}\left[T_{r^{\prime}, 1}\right]_{\left(m^{\prime \prime}, n^{\prime} \pm 1\right)} \ldots\right\rangle \\
& =e^{i \frac{b}{2} \partial_{q-1, s}}\left\langle_{(m, n)}\left[T_{q-1, s}\right]_{\left(m, n^{\prime}\right)}\left[T_{r, 1}\right]_{\left(m^{\prime}, n^{\prime}\right)}\left[T_{r^{\prime}, 1}\right]_{\left(m^{\prime \prime}, n^{\prime}\right)} \ldots\right\rangle .
\end{aligned}
$$

Using these definitions, we get the difference equation satisfied by (91)

$$
\begin{aligned}
& \left(\gamma \widetilde{M}_{3} \sinh \left(b \partial_{\sigma^{\prime}}\right) e^{i \frac{b}{2} \partial_{q-1, s}}-i \sqrt{\mu} \beta \sin ^{2}\left(\pi b^{2}\right) \sinh \left(\pi b \sigma^{\prime}\right) \widetilde{M}_{2} e^{-i \frac{b}{2} \partial_{r, 1}}\right. \\
& \left.-\sqrt{\mu} \tau \sin \left(\pi b^{2}\right) \widetilde{M}_{4}\left\{h\left(\sigma, \sigma^{\prime}\right) \sinh \left(b \partial_{\sigma^{\prime}}\right)+i u\left(\sigma, \sigma^{\prime}\right) \cosh \left(b \partial_{\sigma^{\prime}}\right)\right\} e^{-i \frac{b}{2} \partial_{q-1, s}}\right) \\
& \times\left\langle_{(m, n)}^{\sigma}\left[T_{q-1, s}\right]_{\left(m, n^{\prime}\right)}^{\sigma^{\prime}}\left[T_{r, 1}\right]_{\left(m^{\prime}, n^{\prime}\right)}^{\sigma^{\prime \prime}}\left[T_{r^{\prime}, 1}\right]_{\left(m^{\prime \prime}, n^{\prime}\right)}^{\sigma^{\prime \prime \prime}} \cdots\right\rangle=0,
\end{aligned}
$$

where

$$
\begin{aligned}
& h\left(\sigma, \sigma^{\prime}\right)=\cosh \pi b \sigma^{\prime} \cos \pi b^{2}(1+s)-(-1)^{q} \cosh \pi b \sigma, \\
& u\left(\sigma, \sigma^{\prime}\right)=\sinh \pi b \sigma^{\prime} \sin \pi b^{2}(1+s) .
\end{aligned}
$$

\section{Discussion}

In this work we have focussed exclusively on worldsheet methods to obtain the various results. Naturally one would like to derive them using the dual matrix models - in particular, in order to go beyond the perturbative definition of minimal open string theory. The matrix models in question here are the hermitian two-matrix models, with polynomial potentials of degree $p$ and $q$ in the two matrices $X$ and $Y$ respectively [37].

A Riemann surface $\mathcal{M}_{p, q}$ arises in describing the classical target space of minimal string theory as the moduli space of FZZT branes [14]. This Riemann surface has a complicated sheeted structure determined by the values of $p$ and $q$. The coordinates $x$ and $y$ parametrizing the eigenvalue planes of $X$ and $Y$ can be identified with $\mu_{B}$ and its dual $\tilde{\mu}_{B}$ under $b \rightarrow 1 / b$. The space $\mathcal{M}_{p, q}$ is a $q$-sheeted cover of the $x$ plane and a $p$-sheeted cover of the $y$ plane; $\sigma$ is a uniformizing coordinate for the surface. Asymptotically, for large $x, y$, the shifts $\sigma \rightarrow \sigma+i b$ and $\sigma \rightarrow \sigma+i / b$ are deck transformations taking one 
between the various sheets. Thus the relation (78) equating matter boundary states at fixed $\sigma$ to a sum over states at different values of $\sigma$ tells us that information about the matter states is encoded nonlocally on the surface $\mathcal{M}_{p, q}$ (although for example $|\sigma ; 1, k\rangle$ lives on the points over a single value of $y$, and $|\sigma ; j, 1\rangle$ sits over a single $x$ ). Microscopically, minimal models are described as lattice statistical models with degrees of freedom taking values in a Dynkin diagram [8]. The matter boundary conditions $(1, k)$ describe a Dirichlet boundary condition at a fixed location on the Dynkin diagram [38], which thus appears to be encoded in the sheets of $\mathcal{M}_{p, q}$. Interestingly, this structure seems to be deformed, and disappear altogether, as one passes to the strong coupling region at small $x, y$. Moreover, as argued in [39], the quantum target space turns out to be very different since the FZZT brane correlators are entire functions of $\mu_{B}$. The quantum target space reduces to just the complex plane, and the structure of the Dynkin diagram is less apparent.

It would be helpful to deduce the various results above using matrix models: for example, the relation among boundary states (63). An understanding of the boundary ground ring in matrix models is definitely going to be useful in elucidating the relation between the worldsheet formulation and the nonperturbative formulation given by the matrix model. Also in evaluating correlators in matrix models, one has to know how to encode the matter boundary conditions. The boundary states of the worldsheet theory are labelled by both $\sigma$, which parametrizes the eigenvalue plane of the matrices; and by the matter boundary conditions $(m, n)$. So far, there is no direct understanding of these labels in the matrix model.

It is conceivable that the matter boundary conditions might be related to the choice of the contours of integration on the eigenvalue planes. One can deform the contours to start and end at infinity in such a way that the integral converges. There exists such a choice of contours $[40,41]$ which splits the eigenvalue planes into angular sectors. In fact there are $p-1$ and $q-1$ independent such contours in the $x$ and $y$ planes respectively: the same number as the choices of $(1, s)$ and $(r, 1)$ matter boundary conditions. Considering the Hilbert-Laplace transform of operators along these contours [41] might be related to the choice of matter boundary conditions. A change of basis from these contours to the steepest descent contours as mentioned in [41] may be a natural choice for specifying the matter boundary conditions. It would be interesting to understand concretely how the matter boundary conditions arise from the two-matrix model.

Another issue which we have not addressed concerns the structure of the boundary 
ground ring itself. The bulk ground ring is generated by the bulk $\mathcal{O}_{1,2}$ and $\mathcal{O}_{2,1}$ operators, subject to the relations

$$
U_{q-1}\left(\mathcal{O}_{1,2}\right)=U_{p-1}\left(\mathcal{O}_{2,1}\right)=0
$$

The boundary ground ring is similarly generated by the boundary $\mathcal{O}_{1,2}$ and $\mathcal{O}_{2,1}$ operators, since by fusion these operators generate any $\mathcal{O}_{r, s}$ in their products; however, the ring algebra and relations have not been worked out in this case. The multiplication table is not as simple as in the bulk case; for instance, the product of $\mathcal{O}_{1,2}$ with itself to make the identity depends only on $\mu_{B}$, while the product of $\mathcal{O}_{1,3}$ with itself to make the identity depends on both $\mu$ and $\mu_{B}$. The fact that the multiplication table depends on $\mu_{B}$ means that the whole structure varies as one moves around on the Riemann surface $\mathcal{M}_{p, q}$. Furthermore, the boundary ground ring operators are endomorphisms in the space of open string boundary conditions.

Thus there is room for non-commutative structure, and indeed the product $\mathcal{O}_{1,2} \mathcal{O}_{2,1}$ differs from the product $\mathcal{O}_{2,1} \mathcal{O}_{1,2}$ since they are determined by distinct fusion coefficients. We leave the characterization of the boundary ground ring in terms of generators, relations, etc., to future work.

\section{Acknowledgements}

The work of A. B. is supported in part by NSF Grant No. PHY-0204608, and the work of E. M. is supported in part by DOE grant DE-FG02-90ER40560.

\section{References}

[1] B. H. Lian and G. J. Zuckerman, "New selection rules and physical states in 2-D gravity: Conformal gauge," Phys. Lett. B254 (1991) 417-423.

[2] E. Witten, "Ground ring of two-dimensional string theory," Nucl. Phys. B373 (1992) 187-213, hep-th/9108004.

[3] T. L. Curtright and C. B. Thorn, "Conformally Invariant Quantization of the Liouville Theory," Phys. Rev. Lett. 48 (1982) 1309.

[4] A. A. Belavin, A. M. Polyakov, and A. B. Zamolodchikov, "Infinite conformal symmetry in two-dimensional quantum field theory," Nucl. Phys. B241 (1984) $333-380$. 
[5] N. Seiberg, "Notes on quantum Liouville theory and quantum gravity," Prog. Theor. Phys. Suppl. 102 (1990) 319-349.

[6] J. Polchinski, "Remarks on the Liouville Field Theory,". Presented at Strings '90 Conf., College Station, TX, Mar 12-17, 1990.

[7] I. K. Kostov, "Boundary correlators in 2D quantum gravity: Liouville versus discrete approach," Nucl. Phys. B658 (2003) 397-416, hep-th/0212194.

[8] P. Di Francesco, P. Mathieu, and D. Senechal, "Conformal field theory,". New York, USA: Springer (1997) $890 \mathrm{p}$.

[9] J. L. Cardy, "Boundary Conditions, Fusion Rules and the Verlinde Formula," Nucl. Phys. B324 (1989) 581.

[10] V. Fateev, A. B. Zamolodchikov, and A. B. Zamolodchikov, "Boundary Liouville field theory. I: Boundary state and boundary two-point function," hep-th/0001012.

[11] J. L. Cardy, "Effect of Boundary Conditions on the Operator Content of two-dimensional Conformally Invariant Theories," Nucl. Phys. B275 (1986) 200-218.

[12] B. Ponsot and J. Teschner, "Boundary Liouville field theory: Boundary three point function," Nucl. Phys. B622 (2002) 309-327, hep-th/0110244.

[13] M. Bershadsky and D. Kutasov, "Scattering of open and closed strings in (1+1)-dimensions," Nucl. Phys. B382 (1992) 213-228, hep-th/9204049.

[14] N. Seiberg and D. Shih, "Branes, rings and matrix models in minimal (super)string theory," JHEP 02 (2004) 021, hep-th/0312170.

[15] I. K. Kostov, "Boundary ground ring in 2D string theory," Nucl. Phys. B689 (2004) 3-36, hep-th/0312301.

[16] J. Teschner, "On the Liouville three point function," Phys. Lett. B363 (1995) 65-70, hep-th/9507109.

[17] K. Hosomichi, "Bulk-boundary propagator in Liouville theory on a disc," JHEP 11 (2001) 044, hep-th/0108093. 
[18] B. Ponsot, "Liouville theory on the pseudosphere: Bulk-boundary structure constant," Phys. Lett. B588 (2004) 105-110, hep-th/0309211.

[19] D. C. Lewellen, "Sewing constraints for conformal field theories on surfaces with boundaries," Nucl. Phys. B372 (1992) 654-682.

[20] I. Runkel, "Boundary structure constants for the A-series Virasoro minimal models," Nucl. Phys. B549 (1999) 563-578, hep-th/9811178.

[21] I. Runkel, "Structure constants for the D-series Virasoro minimal models," Nucl. Phys. B579 (2000) 561-589, hep-th/9908046.

[22] R. E. Behrend, P. A. Pearce, V. B. Petkova, and J.-B. Zuber, "Boundary conditions in rational conformal field theories," Nucl. Phys. B570 (2000) 525-589, hep-th/9908036.

[23] G. Felder, J. Frohlich, J. Fuchs, and C. Schweigert, "Correlation functions and boundary conditions in RCFT and three-dimensional topology," Compos. Math. 131 (2002) 189-237, hep-th/9912239.

[24] G. W. Moore and N. Seiberg, "Classical and Quantum Conformal Field Theory," Commun. Math. Phys. 123 (1989) 177.

[25] G. W. Moore and N. Seiberg, "Lectures on RCFT,". Presented at Trieste Spring School 1989, published in Trieste Superstrings 1989:1-129, also in Banff NATO ASI 1989:263-362.

[26] L. Alvarez-Gaume, C. Gomez, and G. Sierra, "Quantum Group Interpretation of some Conformal Field Theories," Phys. Lett. B220 (1989) 142.

[27] L. Alvarez-Gaume, C. Gomez, and G. Sierra, "Duality and Quantum Groups," Nucl. Phys. B330 (1990) 347.

[28] A. N. Kirillov and N. Y. Reshetikhin, "Representations of the Algebra $U_{q}(s l(2))$, q-orthogonal Polynomials and Invariants of Links," New Developments in the Theory of Knots, T. Kohno ed, World. Sc. (1990) 202. 
[29] B. L. Feigin and D. B. Fuks, "Invariant skew symmetric differential operators on the line and verma modules over the Virasoro algebra," Funct. Anal. Appl. 16 (1982) $114-126$.

[30] V. S. Dotsenko and V. A. Fateev, "Conformal algebra and multipoint correlation functions in 2D statistical models," Nucl. Phys. B240 (1984) 312.

[31] V. S. Dotsenko and V. A. Fateev, "Four Point Correlation Functions and the Operator Algebra in the two-dimensional Conformal Invariant Theories with the Central Charge $C<1$," Nucl. Phys. B251 (1985) 691.

[32] N. Ishibashi, "The Boundary and Crosscap States in Conformal Field Theories," Mod. Phys. Lett. A4 (1989) 251.

[33] T. Onogi and N. Ishibashi, "Conformal Field Theories on Surfaces with Boundaries and Crosscaps," Mod. Phys. Lett. A4 (1989) 161.

[34] D. Kutasov, E. J. Martinec, and N. Seiberg, "Ground rings and their modules in 2-D gravity with $c \leq 1$ matter," Phys. Lett. B276 (1992) 437-444, hep-th/9111048.

[35] I. R. Klebanov, "Ward identities in two-dimensional string theory," Mod. Phys. Lett. A7 (1992) 723-732, hep-th/9201005.

[36] S. Kachru, "Quantum rings and recursion relations in 2-D quantum gravity," Mod. Phys. Lett. A7 (1992) 1419-1426, hep-th/9201072.

[37] J. M. Daul, V. A. Kazakov, and I. K. Kostov, "Rational theories of 2-D gravity from the two matrix model," Nucl. Phys. B409 (1993) 311-338, hep-th/9303093.

[38] H. Saleur and M. Bauer, "On some relations between local height probabilities and conformal invariance," Nucl. Phys. B320 (1989) 591.

[39] J. Maldacena, G. W. Moore, N. Seiberg, and D. Shih, "Exact vs. semiclassical target space of the minimal string," JHEP 10 (2004) 020, hep-th/0408039.

[40] M. Bertola, B. Eynard, and J. P. Harnad, "Duality, Biorthogonal Polynomials and Multi-Matrix Models," Commun. Math. Phys. 229 (2002) 73-120, nlin.si/0108049. 
[41] M. Bertola, B. Eynard, and J. P. Harnad, "Differential systems for biorthogonal polynomials appearing in 2-matrix models and the associated Riemann-Hilbert problem," Commun. Math. Phys. 243 (2003) 193-240, nlin.si/0208002. 